\title{
In-situ conductivity and Seebeck measurements of highly efficient n-dopants in fullerene $\mathrm{C}_{60}$
}

\author{
Torben Menke, Debdutta Ray, Jan Meiss, Karl Leo, a) and Moritz Riede \\ Institut für Angewandte Photophysik, Technische Universität Dresden, 01062 Dresden, Germany
}

(Received 9 January 2012; accepted 9 February 2012; published online 29 February 2012)

\begin{abstract}
We present two organic dimetal complexes $\mathrm{Cr}_{2}(\mathrm{hpp})_{4}$ and $\mathrm{W}_{2}(\mathrm{hpp})_{4}$ as n-dopants investigated in the model system of fullerene $\mathrm{C}_{60}$ for the application in organic electronic devices. Conductivity and Seebeck measurements on doped layers are carried out in vacuum at different doping concentrations and various substrate temperatures to compare the two dopants. Very high conductivities of up to $4 \mathrm{~S} / \mathrm{cm}$ are achieved for both organic dopants. The thermal activation energy of the conductivity as well as the measured Seebeck coefficient are found to decrease with increasing doping concentration, indicating a shift of the Fermi level towards the electron transport level of the n-doped $\mathrm{C}_{60}$. $(\mathrm{C} 2012$ American Institute of Physics. [http://dx.doi.org/10.1063/1.3689778]
\end{abstract}

Organic optoelectronic devices based on small molecules are advancing towards commercial maturity, and new materials and concepts have resulted in high efficiencies of small molecule-based organic light-emitting diodes $(\mathrm{OLED})^{1}$ and solar cells. ${ }^{2}$

Many of the high performance devices employ the pinconcept. ${ }^{3}$ Here, $\mathrm{n}$ and $\mathrm{p}$ doped organic layers serve as highly conductive charge transport layers that open the pathway to quasi-ohmic contacts between the photoactive layers and the electrodes. This is made possible as doping allows for the controlling of the energetic position of the Fermi level, and therefore, the charge carrier injection barriers can be minimized in the co-deposited layers. In this publication, we focus on n-type doping, which can be achieved either by incorporating metal atoms (e.g., alkali metals of groups 1 and 2 like Cs (Refs. 4 and 5)) or by organic molecules (Refs. 6 and 7) which act as electron donors. The latter is preferred since the small metal atoms can lead to decreased device efficiency and lifetimes due to metal diffusion. ${ }^{3}$ Molecular doping is performed by simultaneous co-evaporation of an organic matrix and a few molar percentage of organic dopant. The study of doping in organic semiconductors is still in an early stage, and there are still many open questions regarding the exact mechanism of doping.

In this letter, we present our work on n-doped fullerene $\mathrm{C}_{60}$ layers, comparing two n-dopants $\mathrm{Cr}_{2}(\mathrm{hpp})_{4}$ and $\mathrm{W}_{2}(\mathrm{hpp})_{4}$. For this, in-situ conductivity and Seebeck measurements in vacuum are performed, and the influence of the doping ratio and of a temperature variation on these properties is compared. As expected, with increasing doping concentration, we can find an increasing conductivity for both dopants, which saturates at around $4 \mathrm{~S} / \mathrm{cm}\left(\mathrm{T}=25^{\circ} \mathrm{C}\right)$, which is well above the highest values reported for $\mathrm{C}_{60}$ doped by organic compounds. ${ }^{8-10}$

With the Seebeck studies, we can directly measure the position of the Fermi level with respect to the transport level of the differently doped layers. We find a decreasing Seebeck coefficient, indicating a shift of the Fermi level towards

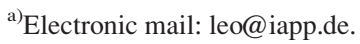

the electron transport state, for increasing doping concentrations. A similar decrease is found for the thermal activation energy of the conductivity.

As host material, we use sublimated $\mathrm{C}_{60}$ (purchased from CreaPhys $\mathrm{GmbH}$, Germany; used as received). The dopants (purchased from Novaled AG, Germany) are dimetal complexes of chromium or tungsten with the anion of 1,3,4,6,7,8-hexahydro-2 H-pyrimido[1,2-a]pyrimidine (hpp), forming $\mathrm{Cr}_{2}(\mathrm{hpp})_{4}$ and $\mathrm{W}_{2}(\mathrm{hpp})_{4}$. These compounds were first published by Cotton et al., ${ }^{11,12}$ and their use in OLEDs has been described by Novaled. ${ }^{13,14}$ They exhibit very low ionization potentials, $\mathrm{W}_{2}(\mathrm{hpp})_{4}$ being even shallower than $\mathrm{Cs}(\mathrm{IP}=3.9 \mathrm{eV})$, the least electronegative stable element. As these compounds easily oxidize in air, they have to be handled in inert gas atmosphere or vacuum. The molar weight of the materials $\mathrm{C}_{60}, \mathrm{Cr}_{2}(\mathrm{hpp})_{4}$, and $\mathrm{W}_{2}(\mathrm{hpp})_{4}$, needed for the calculation of the molar ratios (MRs) in the doped layers, are 720.6, 656.8, and $920.4 \mathrm{~g} / \mathrm{mol}$, respectively. Their molecular structures are shown in Fig. 1.

The experimental setup is described in detail elsewhere. ${ }^{6}$ A high vacuum chamber (base pressure $10^{-4}-10^{-5} \mathrm{~Pa}$ ) is used to prepare and measure the samples in-situ. Matrix and dopant are co-evaporated onto a glass substrate, which has been pre-structured with two parallel Au contacts with $5 \mathrm{~mm}$ inter-finger distance and a thickness of $40 \mathrm{~nm}$.

The $30 \mathrm{~nm}$ thick doped layers are deposited onto the substrate at a rate of $0.15 \AA / \mathrm{s}$ with a substrate temperature of $T_{\text {sub }}=25^{\circ} \mathrm{C}$. The evaporation rates of matrix and dopant, and thus the doping concentration, are monitored independently using two quartz crystal monitors.

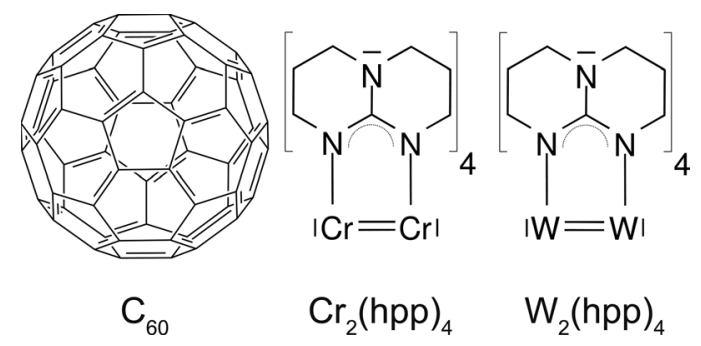

FIG. 1. Molecular structures of the materials used. 
The current between the two Au fingers is found to vary linearly and symmetrically with the voltage in the range of $-10 \mathrm{~V}$ to $+10 \mathrm{~V}$ for all samples. Due to the low field, contact resistance is expected to be negligible compared to bulk resistance in a planar sample with $5 \mathrm{~mm}$ inter-electrode width. Furthermore, the doping ensures a quasi-ohmic contact between organic material and metal electrode. ${ }^{15}$ Therefore, the current is not injection limited in the investigated regime.

In-situ measurements of conductivity and thermovoltage (Seebeck coefficient) are performed using a Keithley 236 source measure unit. Heating is applied from the back of the substrate by two independently heated copper blocks. The temperature of the sample $T_{\mathrm{m}}$ is controlled by a two-channel Eurotherm 3504 PID controller and two PT1000 resistance based temperature sensors that are glued onto the substrate at thermally equivalent positions to the contacts, insulated from the organic layer. This allows for substrate temperatures in the range of $20-110^{\circ} \mathrm{C}$.

After preparation, the samples are slowly heated up and annealed for $20 \mathrm{~min}$ at $100^{\circ} \mathrm{C}$ as the annealing can increase the conductivity of n-doped layers by a factor of 3-5 and further allows for stable measurement conditions after reaching saturation, as proposed by Nollau et al. ${ }^{6}$ We find both dopants to be stable even at $T_{\mathrm{m}}=100^{\circ} \mathrm{C}$, and no degradation of the conductivity was observed. For lowly doped samples, we observe that the conductivity increases due to the annealing, while for highly doped samples, a decrease is found. The increase might be attributed to a removal of oxygen unintentionally built into the film during deposition, while the decrease might be explained by diffusion and clustering of dopant molecules.

The conductivity at a temperature $T_{\mathrm{m}}$ is determined by applying a voltage of $V_{\mathrm{b}}=1 \mathrm{~V}$ and averaging the measured current for $2 \mathrm{~min}$ in order to compensate for statistical noise. For the Seebeck measurement at any temperature $T_{\mathrm{m}}$, a difference of $\Delta T=5 \mathrm{~K}$ is applied, as the two contacts are heated to temperatures $T_{\mathrm{m}}+2.5 \mathrm{~K}$ and $T_{\mathrm{m}}-2.5 \mathrm{~K}$, respectively. The measured thermovoltage $V_{\mathrm{s}}$ is averaged for several minutes, followed by a measurement at swapped temperatures to exclude systematic errors. The resulting Seebeck coefficient $\left(S=V_{\mathrm{s}} / \Delta T\right)$ is the average of the two measurements. Before and after each Seebeck measurement, the conductivity $\sigma\left(T_{\mathrm{m}}\right)$ is determined and the two values are as well averaged.

Using ultraviolet photoelectron spectroscopy (UPS), the IPs for $\mathrm{Cr}_{2}(\mathrm{hpp})_{4}$ and $\mathrm{W}_{2}(\mathrm{hpp})_{4}$ are determined to be $3.95 \pm$ $0.13 \mathrm{eV}$ and $2.68 \pm 0.13 \mathrm{eV}$, respectively, both smaller than the electron affinity (EA) of $\mathrm{C}_{60}(4.0 \pm 0.3 \mathrm{eV}$ (Ref. 16)). Hence, efficient charge transfer and, therefore, good doping are expected, especially for $\mathrm{W}_{2}(\mathrm{hpp})_{4}$, because its IP is even shallower than the very efficient molecular dopant decamethylcobaltocene (DMC) $(\mathrm{IP}=3.3 \mathrm{eV}$ (Ref. 17)).

We study a variation of different doping concentrations ranging from MR of $0.002-0.4$ and $T_{\mathrm{m}}=25-70{ }^{\circ} \mathrm{C}$. Figure 2 shows the conductivity $\sigma$ of the n-doped $\mathrm{C}_{60}$ layers versus doping concentration in a double-logarithmic plot for $T_{\mathrm{m}}=25^{\circ} \mathrm{C}$ and $40^{\circ} \mathrm{C}$ (which is a typical operation temperature for organic semiconductor devices). For both dopants, a strong increase of the conductivity from around 0.1 to $4 \mathrm{~S} / \mathrm{cm}$ with increasing doping is observed. In comparison, undoped

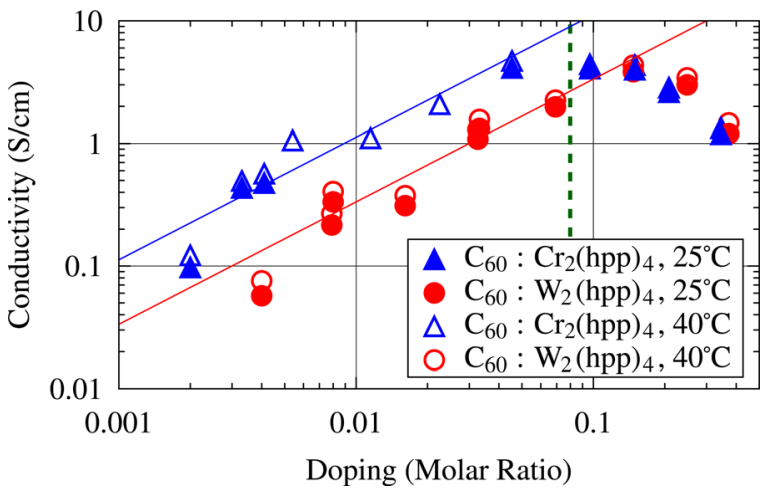

FIG. 2. (Color online) Conductivity $\sigma$ of $30 \mathrm{~nm}$ n-doped $\mathrm{C}_{60}$ layers versus doping concentration. Up to doping concentrations of $M R=0.08$ (dashed line), a linear relation between doping and conductivity is found. The solid lines representing a slope of 1.0 as a guide for the eye.

$\mathrm{C}_{60}$ has a conductivity in the order of $10^{-8} \mathrm{~S} / \mathrm{cm} .{ }^{9} \mathrm{Up}$ to doping concentrations of MR $=0.08$ (dashed line in Fig. 2), a linear relation between doping and conductivity is found, as the lines representing a slope of 1.0 show. In this doping regime, each additional dopant molecule contributes identically to the increase of conductivity. This is not always the case as earlier studies on organic dopants showed. ${ }^{18,19}$ At doping concentrations above $\mathrm{MR}=0.08$, both materials show a saturation of the conductivity. A possible explanation could be a drop in the electron mobility due to a disturbance of the morphology when more than 8 dopants molecules per 100 molecules of $\mathrm{C}_{60}$ are introduced into the layer. ${ }^{20,21}$

In contrast to what would be expected from the UPS measured IP values, using $\mathrm{Cr}_{2}(\mathrm{hpp})_{4}(\mathrm{IP}=3.95 \pm 0.13 \mathrm{eV})$ leads to a more efficient doping of $\mathrm{C}_{60}(\mathrm{EA}=4.0 \pm 0.3 \mathrm{eV})$ compared to $\mathrm{W}_{2}(\mathrm{hpp})_{4}(\mathrm{IP}=2.68 \pm 0.13 \mathrm{eV})$. Assuming that there is no renormalization of molecular levels on formation of an ion, this observation might be explained by morphological effects, or by $\mathrm{W}_{2}(\mathrm{hpp})_{4}$ reacting stronger with oxygen contamination being present in the vacuum chamber at a base pressure of $10^{-4}-10^{-5} \mathrm{~Pa}$. Further studies are required to resolve this observation.

In the investigated range of $T_{\mathrm{m}}=25-70^{\circ} \mathrm{C}$, the conductivity $\sigma$ is found to be thermally activated, as shown earlier for doped organic layers, ${ }^{22,23}$ and the data can be fitted with a thermally activated transport relation

$$
\sigma(T) \propto \exp \left(-\frac{E_{\mathrm{Act}, \sigma}}{k_{\mathrm{B}} T}\right),
$$

where $k_{\mathrm{B}}$ is Boltzmann's constant and $E_{\mathrm{Act}, \sigma}$ is the activation energy of the conductivity. The derived activation energies $E_{\mathrm{Act}, \sigma}$ shown in Fig. 3 range from 50 to $160 \mathrm{eV}$ and show a strong dependence on the doping concentration. Up to doping concentrations of $\mathrm{MR}=0.08$, a decrease of $E_{\mathrm{Act}, \sigma}$ with increasing doping concentration is found, as expected from earlier experiments. ${ }^{9,23}$ The observed $E_{\text {Act }, \sigma}$ for both dopants match within experimental scattering and are well below the previously reported value for undoped $\mathrm{C}_{60}$ of around $650 \mathrm{meV} .{ }^{9}$ Highly doped samples of doping concentrations above $\mathrm{MR}=0.08$ show a different behavior of increasing $E_{\mathrm{Act}, \sigma}$ with doping. This is in agreement with the conductivity data shown in Fig. 2. The decrease of $E_{\text {Act }, \sigma}$ with 


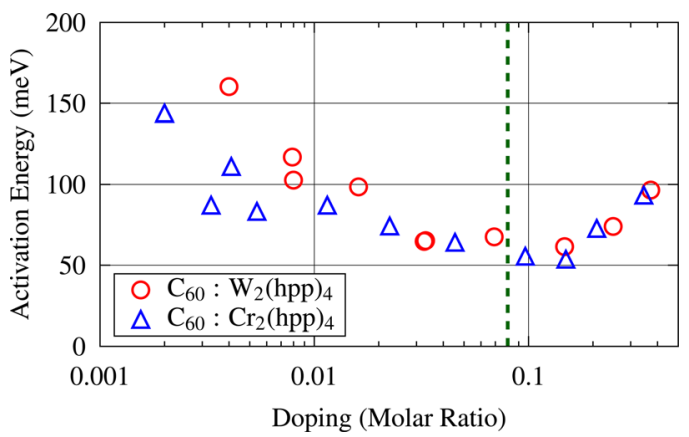

FIG. 3. (Color online) Thermal activation energy of the conductivity $E_{\mathrm{Act}, \sigma}$ versus doping concentration for the two different material systems. The dashed line corresponds to $M R=0.08$, as in Fig. 2 .

increasing MR is attributed to a shift of the Fermi level towards the transport level of $\mathrm{C}_{60}$. This leads to a reduction of the temperature dependence of the charge carrier density $n(T)$, contributing to the conductivity as

$$
\sigma(T)=e \cdot n(T) \cdot \mu(T),
$$

with the elementary charge $e$ and mobility $\mu(T)$, which also contributes to the temperature dependency of $\sigma$ and, hence, $E_{\mathrm{Act}, \sigma}$. The increase of $E_{\mathrm{Act}, \sigma}$ at high doping concentrations might be attributed to the mobility, which is probably due to disturbance in the morphology of the host material by the large percentage of dopant molecules.

The Seebeck measurements (Fig. 4) at $T_{\mathrm{m}}=40^{\circ} \mathrm{C}$ show that the thermo-voltage and, hence, the Seebeck coefficient $S$ are negative in sign. Therefore, for all samples, electrons are the dominating charge carriers, and hole conduction along the dopant molecules is not observed. For both dopants, a decrease of $|S|$ with increasing doping concentration is found. Assuming a Gaussian density of states, the energetic difference $E_{\mathrm{S}}$ of the Fermi level $E_{\mathrm{F}}$ to the transport level $E_{\mathrm{Tr}}$ can be derived from Seebeck measurements ${ }^{24,25}$ via

$$
E_{\mathrm{S}}:=E_{\mathrm{Tr}}-E_{\mathrm{F}}=e \cdot \mathrm{T} \cdot|S| .
$$

This quantity is shown as the right hand y-axis in Fig. 4 for $T_{\mathrm{m}}=40^{\circ} \mathrm{C}$. For both dopants, a decrease of $E_{\mathrm{S}}$ with increasing doping concentration is observed, indicating a shift of the Fermi level towards the transport level. Using MaxwellBoltzmann approximation, it has been shown ${ }^{6}$ that the charge carrier density $n(T)$ is thermally activated by $E_{\mathrm{S}}$. In

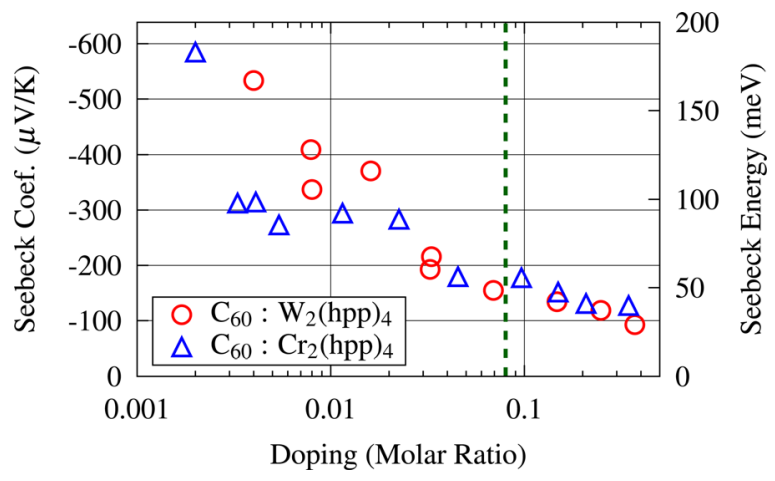

FIG. 4. (Color online) Seebeck coefficient and derived Seebeck energy $E_{\mathrm{S}}$ at $T_{\mathrm{m}}=40^{\circ} \mathrm{C}$. The dashed line corresponds to $M R=0.08$, as in Fig. 2. the case of our highly doped samples, $E_{\mathrm{S}}$ is in the order of $k T$, and therefore, the approximation is not valid, but still a decrease of $E_{\mathrm{S}}$ can be correlated to a decrease of the thermal activation of $n(T)$. We note from Fig. 4 that the Seebeck coefficient trends towards saturation at large doping concentrations. This is expected since the system is degenerately doped at large doping densities, and the Fermi level gets pinned near the transport level edge. Considering the continuing decrease in the Seebeck coefficient even after the 0.08 doping mark, we can verify that the final increase of $E_{\mathrm{Act}, \sigma}$ and decrease of conductivity at high doping concentrations must be due to changes in the mobility of the layer.

We show that both $\mathrm{Cr}_{2}(\mathrm{hpp})_{4}$ and $\mathrm{W}_{2}(\mathrm{hpp})_{4}$ are exceptionally well suited for $\mathrm{n}$-doping of $\mathrm{C}_{60}$, with extremely high conductivities up to $4 \mathrm{~S} / \mathrm{cm}$ at $40^{\circ} \mathrm{C}$. A linear correlation between doping concentration (up to $\mathrm{MR}=0.08$ ) and conductivity is found for both dopants. For low doping concentrations, the $\mathrm{Cr}_{2}(\mathrm{hpp})_{4}$ leads to better conductivities than the $\mathrm{W}_{2}(\mathrm{hpp})_{4}$. Furthermore, with increasing doping concentration, the activation energy $E_{\mathrm{Act}, \sigma}$ drops from $150 \mathrm{meV}$ to $50 \mathrm{meV}$ and the Seebeck measurements show the same trend for the energetic difference between the Fermi-level and the transport level. For very high doping rates, an increase in the activation energy is observed, while this is not present in the Seebeck data. This can be attributed to a change in film morphology, which affects the mobility negatively.

This work is supported by the German Federal Ministry of Education and Research (BMBF) in the framework of the Project No. 13N9720 (OPEG). The authors acknowledge Novaled AG, Germany, for providing the dopants.

${ }^{1}$ S. Reineke, F. Lindner, G. Schwartz, N. Seidler, K. Walzer, B. Lüssem, and K. Leo, Nature 459, 234 (2009).

${ }^{2}$ M. A. Green, K. Emery, Y. Hishikawa, and W. Warta, Prog. Photovoltaics 19, 84 (2011).

${ }^{3}$ K. Walzer, B. Maennig, M. Pfeiffer, and K. Leo, Chem. Rev. 107, 1233 (2007).

${ }^{4}$ R. C. Haddon, A. F. Hebard, M. J. Rosseinsky, D. W. Murphy, S. J. Duclos, K. B. Lyons, B. Miller, J. M. Rosamilia, R. M. Fleming, A. R. Kortan et al., Nature 350, 320 (1991).

${ }^{5}$ M. Ramsey, D. Steinmüller, and F. Netzer, Phys. Rev. B 42, 5902 (1990).

${ }^{6}$ A. Nollau, M. Pfeiffer, T. Fritz, and K. Leo, J. Appl. Phys. 87, 4340 (2000).

${ }^{7}$ S. Guo, S. B. Kim, S. K. Mohapatra, Y. Qi, T. Sajoto, A. Kahn, S. R. Marder, and S. Barlow, Adv. Mater. 24, 699 (2012).

${ }^{8}$ F. Li, A. Werner, M. Pfeiffer, K. Leo, and X. Liu, J. Phys. Chem. B 108, 17076 (2004).

${ }^{9}$ F. Li, M. Pfeiffer, A. Werner, K. Harada, K. Leo, N. Hayashi, K. Seki, X. Liu, and X.-D. Dang, J. Appl. Phys. 100, 23716 (2006).

${ }^{10}$ N. Hayashi, K. Kanai, Y. Ouchi, and K. Seki, in Organic Electronics Materials, Devices and Applications (Mater. Res. Soc. Symp. Proc., 2006), Vol. 965, p. 0965-S13-03.

${ }^{11}$ F. A. Cotton, N. E. Gruhn, J. Gu, P. Huang, D. L. Lichtenberger, C. A. Murillo, L. O. Van Dorn, and C. C. Wilkinson, Science (N.Y.) 298, 1971 (2002).

${ }^{12}$ F. A. Cotton, J. P. Donahue, D. L. Lichtenberger, C. A. Murillo, and D. Villagrán, J. Am. Chem. Soc. 127, 10808 (2005).

${ }^{13}$ A. Werner, O. Kühl, S. Gessler, K. Harada, H. Hartmann, A. Grüssing, M. Limmert, and A. Lux, "Use of metal complexes as n-dopants for organic semiconductors and production thereof including their ligands," patent PCT/DE2005/000372 (2005).

${ }^{14} \mathrm{P}$. Wellmann, "Lebensdauer und stabilität elektrisch dotierter OLEDs," Ph.D. dissertation, TU Dresden, 2009.

${ }^{15}$ J. Blochwitz, M. Pfeiffer, T. Fritz, and K. Leo, Appl. Phys. Lett. 73, 729 (1998).

${ }^{16}$ W. Zhao and A. Kahn, J. Appl. Phys. 105, 123711 (2009). 
${ }^{17}$ C. Chan, W. Zhao, S. Barlow, S. Marder, and A. Kahn, Org. Electron. 9, 575 (2008).

${ }^{18}$ B. Maennig, M. Pfeiffer, A. Nollau, X. Zhou, K. Leo, and P. Simon, Phys. Rev. B 64, 195208 (2001).

${ }^{19}$ B. A. Gregg, S.-G. Chen, and R. A. Cormier, Chem. Mater. 16, 4586 (2004).

${ }^{20}$ H. Kleemann, C. Schuenemann, A. A. Zakhidov, M. Riede, B. Lüssem, and K. Leo, Org. Electron. 13, 58 (2012).
${ }^{21}$ K. Harada, M. Sumino, C. Adachi, S. Tanaka, and K. Miyazaki, Appl. Phys. Lett. 96, 253304 (2010).

${ }^{22}$ S. Fujimori, K. Hoshimono, and S. Fujita, Solid State Commun. 89, 437 (1994).

${ }^{23}$ M. Pfeiffer, A. Beyer, T. Fritz, and K. Leo, Appl. Phys. Lett. 73, 3202 (1998).

${ }^{24}$ H. Fritzsche, Solid State Commun. 9, 1813 (1971).

${ }^{25}$ R. Schmechel, J. Appl. Phys. 93, 4653 (2003). 